\title{
A Good Outcome Following Delayed Debridement of Lumbar Paraspinal Abscess after Postoperative Epidural Catheterization: Case Report
}

\author{
Azizul Akram Salim ${ }^{\text {** Dayang Shariza Abang Abdillah', Hong Chun Lim }}$, Joehaimey Johari', \\ Mohd Imran Yusof ${ }^{5}$ \\ ${ }^{1,2,3,4,5}$ Universiti Sains, Malaysia
}

\begin{abstract}
Paraspinal abscess or clinically significant soft tissue infection following epidural analgesia is a rare but serious complication if left untreated. We report a 54-year-old gentleman with underlying chronic diabetes mellitus who had a motor vehicle accident and had undergone several surgeries. A course of steroid was provided for delayed left lower motor neuron facial nerve palsy (House-Brackmann stage 4). The epidural catheter was inserted with a single attempt at the L3/L4 level. One month later, he complained of crippling lower back pain and growing swelling with a punctum. MRI showed a paraspinal abscess with bilateral involvement of the spinae erector. This report is intended to highlight the excellent outcome of surgical treatment for paraspinal abscess despite the late presentation. A high level of suspicion should be taken into account when the patient is immunocompromised, particularly with steroid use and chronic diabetes mellitus. Early detection via MRI, high suspicion index and immediate surgery have extraordinary benefits in managing paraspinal abscesses.
\end{abstract}

Key words: spinal debridement, paraspinal abscess, postoperative epidural abscess, surgical site infection

\section{Introduction}

Epidural anaesthesia is a form of regional anaesthetic widely used for analgesia in surgery involving the trunk and lower limb. ${ }^{1}$ Paraspinal abscess or clinically significant soft-tissue infection following epidural analgesia is a rare complication. $^{2,3}$ Generally, the incidence of post-operative infection at the insertion site is reported as $0.052 \%$ in a prospective study where only nine cases of epidural abscesses were published in a series of 17,372 epidural catheters. ${ }^{3}$

Infections related to epidural catheterization can occur either superficial, deep, or in the

\section{*Correspondence: Azizul Akram Salim \\ E mail: azeck83@gmail.com}

https://orcid.org/0000-0001-8650-3001

Received: $21 / 10 / 2020$

Accepted: 22/8/2021

DOI: https://doi.org/10.4038/slja.v29i2.8701 epidural space. ${ }^{3}$ It may also occur via an exogenous channel such as contaminated equipment or drugs, or from an endogenous source as bacteraemia, which seeds to the insertion site. Although the infection rates are relatively low, complications following this could implicate serious neurological deficit and death.

\section{Case Report}

A 54-year-old gentleman with underlying chronic diabetic mellitus presented following a motor vehicle accident. He sustained cerebral concussion, splenic injury, multiple rib fractures, left scapula and left tibial plateau fractures. His vital signs remained stable. On day 10 of admission, he was diagnosed with delayed left lower motor neuron facial nerve palsy (House-Brackmann stage 4). Prednisolone $20 \mathrm{mg} 8$ hourly was given for three days followed by $25 \mathrm{mg}$ twice a day for another two days.

Steroids were withheld after five days in the absence of clinical improvement. He underwent an endoscopic left facial nerve decompression 
and ossiculoplasty for delayed onset left facial nerve palsy secondary to left temporal bone fracture. Concomitantly, he underwent open reduction and plating of left tibial plateau with iliac bone grafting. He was then given epidural anaesthesia for post-operative pain relief. Epidural catheter was inserted in the first attempt at L3/L4 level under general anaesthesia before extubation in the lateral position under strict aseptic technique. Epidural catheter was removed on day three after optimizing the analgesia.

On post-op day 5 , he developed swelling and redness over the upper lumbar region with a punctum over his back but no pus discharge seen. Clinical examination revealed mild tenderness over the palpable mass, which measured $3 \mathrm{~cm} \times 3 \mathrm{~cm}$ with no neurological deficits noted. Ultrasonography of lower back was done and reported a superficial collection extending from supraspinous ligament to interspinous ligament. He was treated with intravenous cloxacillin $1 \mathrm{~g} 6$ hourly for four days and discharged home with oral antibiotic following clinical improvement. One month later, he presented again complaining of debilitating lower back pain and increasing swelling with the presence of a punctum.

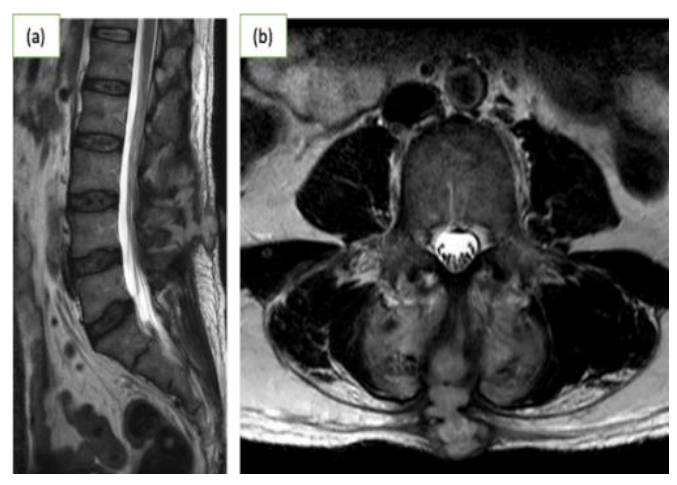

Figure 1. (a) MRI sagittal and (b) axial views showing involvement of bilateral erector spinae and spinalis muscles and severe destruction of L3 and L4 spinous processes. No epidural collection of fluids.

He was unable to maintain upright posture due to the pain. MRI was done and showed paraspinal abscess with bilateral erector spinae muscle involvement (Fig. 1). There was no epidural or intrathecal involvement. Wound debridement of L3/L4 paraspinal muscles and removal of L3/L4 spinous processes, interspinous and supraspinous ligaments were done (Fig. 2). A liquified abscess of 10cc was evacuated. Culture revealed Pseudomonas aeruginosa sensitive to piperacillintazobactam and ciprofloxacin. He was given intravenous piperacillin-tazobactam $2 \mathrm{~g}$ daily for two days and discharged with ciprofloxacin for another six weeks. Post-operatively, there was no back pain and he was able to ambulate independently.

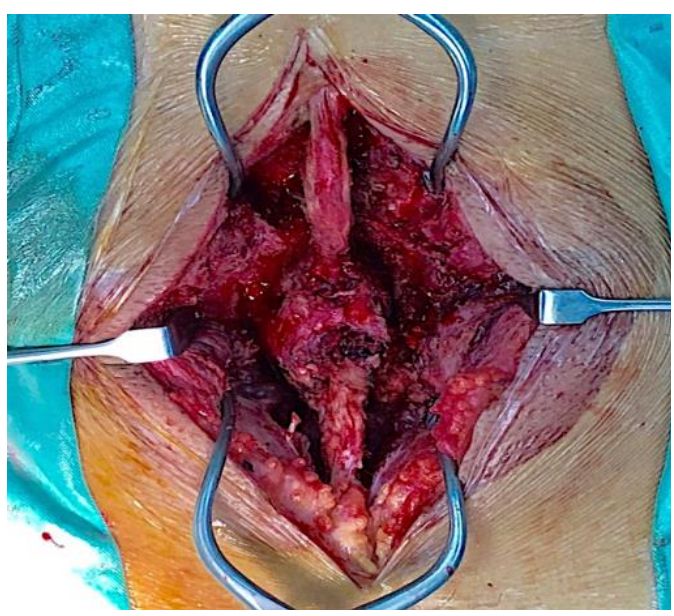

Figure 2. Intraoperative image revealing severe destruction of L3 and L4 spinous processes and intramuscular collection of pus in bilateral erector spinae.

\section{Discussion}

Paraspinal abscess or soft-tissue infection surrounding the paraspinal region following epidural analgesia is a rare encounter. ${ }^{3}$ Despite its rare occurrence, any types of infections related to epidural techniques are potentially detrimental to the patient due to the risk of progression to epidural abscess over a variable time interval.

Back pain is usually the first presenting symptom of epidural analgesia-related infection. Raj \& Foy reported a case of a Mycobacterium tuberculosis paraspinal abscess, presenting 19 days postpartum following the insertion of a labour epidural and assisted vaginal delivery, where severe postpartum back pain was a presenting symptom. ${ }^{4}$ A similar presentation was reported 
by $\mathrm{J} S$ Hill et al. ${ }^{2}$ Based on previously reported cases, the possibility of an epidural or paraspinal abscess was considered. There are many pre-existing pathologies making patients potentially liable to epidural-related infections, such as diabetes, malnutrition, or immunecompromised conditions. ${ }^{3}$ Most of the studies reported recognizable risk factors for infections such as diabetes mellitus, chronic renal failure and immunosuppression secondary to malignancies or steroid administration. Additionally, there should be a low threshold for MRI scan for patients with high degree of suspicion for epidural insertion related complications. ${ }^{5}$ This will enable prompt diagnosis and treatment with a reduced likelihood of permanent neurological sequelae.

Surgical treatment of paraspinal abscess would have a good outcome even if they presented late. A high index of suspicion must be taken into account if the patient is immunocompromised, especially with steroid intake and diabetes mellitus. The number of attempts of epidural catheterization needs to be minimized to reduce the incidence of infection. The insertion site should be covered with a transparent occlusive dressing to inspect the site daily.

\section{Conclusion}

Epidural catheter-related infections have a very significant impact, especially in the immunocompromised group, even though it is uncommon. In our case, the recognizable risk factors were uncontrolled diabetes mellitus and corticosteroids. Serious complications may arise if the condition is left untreated. Hence, early detection via MRI, high-index of suspicion in high-risk group and immediate surgery will have extraordinary benefits in managing paraspinal abscesses.

\section{References}

1. Dawson SJ. Epidural catheter infections. $J$ Hosp Infect. 2001; 47(1):3-8.

2. Hill JS, Hughes EW, Robertson PA. A Staphylococcus aureus paraspinal abscess associated with epidural analgesia in labour. Anaesthesia. 2001; 56(9):873-8.

3. Xue X, Song J, Liang Q, Qin J. Bacterial infection in deep paraspinal muscles in a parturient following epidural analgesia: a case report and literature review: a CAREcompliant article. Medicine (Baltimore). 2015; 94(50).

4. Raj V, Foy J. Paraspinal abscess associated with epidural in labour. Anaesth Intensive Care. 1998; 26(4):424-6.

5. Phillips JMG, Stedeford JC, Hartsilver E, Roberts C. Epidural abscess complicating insertion of epidural catheters. Br J Anaesth. 2002; 89(5):778-82. 